\title{
ADS-B over Satellite
}

\section{Global Air Traffic Surveillance from Space}

\author{
K. Werner \\ Institute of Flight Guidance \\ German Aerospace Center \\ Braunschweig, Germany \\ Email: klaus.werner@dlr.de
}

\author{
J. Bredemeyer \\ FCS Flight Calibration Services \\ Braunschweig, Germany \\ Email: brd@flightcalibration.de
}

\author{
T. Delovski \\ Institute of Space Systems \\ German Aerospace Center \\ Bremen, Germany \\ Email: toni.delovski@dlr.de
}

\begin{abstract}
The steadily increasing air traffic in particular on transoceanic routes requires extending controlled airspace to those regions not yet covered by ground based surveillance. In this paper the authors present the world's first in-orbit demonstration of a space based ADS-B system, hosted on the ESA satellite PROBA-V.
\end{abstract}

Keywords-ADS-B, ATC, PROBA-V, SATELLITE

\section{INTRODUCTION}

Air traffic surveillance as applied in controlled airspace is nowadays mostly based on radar ground stations using Primary Surveillance Radar (PSR), Secondary Surveillance Radar (SSR) or Mode-S radars. Air traffic regions with high traffic density and en-route separation minima of 5 NM require seamless and continuous surveillance, which is ensured by a complex infrastructure of radar stations, radar networks and surveillance data processing systems. This surveillance infrastructure as implemented in structurally well-developed continental areas like Europe, the U.S. or certain regions in Asia, provides comprehensive situation awareness to the controllers in the air traffic control centers as required for a safe and efficient air traffic management.

For several years a further surveillance technology has been developed and introduced in certain flight regions: ADS-B (Automatic Dependent Surveillance - Broadcast) as implemented in modern Mode-S transponders on board of aircraft transmits periodically the flight position and other information by Extended Squitter messages (1090ES) on the $1090 \mathrm{MHz}$ SSR-Mode-S downlink frequency (ADS-B Out). As ADS-B receiving ground stations are less complex and costly than radar stations, they will complement or even replace radar stations in the future, being integrated in the existing surveillance infrastructure.

The European ADS-B Implementing Rule mandates that new aircraft heavier than $5700 \mathrm{~kg}$ or faster than 250 knots will be equipped with ADS-B-Out from 2015 on, and for existing aircraft a retrofit from end of 2017 on. In 2020 ADS-B surveillance shall become operational. Similar mandates exist for some Asian regions and the U.S., whilst in Australia ADSB-Out was required from end of 2012 on.

On the other hand most regions of the world are uncontrolled airspace, since in oceanic, polar or mountainous regions or underdeveloped continental areas the installation of ground based surveillance systems is either technically or economically impossible. In these so-called Non-Radar Airspaces (NRA) surveillance is applied procedurally, which means that the pilot issues position reports via aircraft radio when certain waypoints have been reached. Modern aircraft are also equipped with ADS-C (Automatic Dependent Surveillance - Contract), a point-to-point data link connection based on FANS1/A equipment and Satcom or HFDL data link. Due to limited bandwidth and service costs the system transmits the flight position and other information only every 15 minutes. In both cases no seamless and continuous flight surveillance is possible, with the consequence of separation distances of $50-$ $80 \mathrm{NM}$ due to safety reasons.

In the case of a flight accident the situation may be more problematic, when search and rescue measures have to be performed without knowledge of the crash site position. After the accident of the AF447 flight from Rio de Janeiro to Paris in the south Atlantic it took five days to locate the crash site, and two more years to recover the black box.

In order to avoid misunderstandings it must be stated clearly and with regard to a recent fatal accident, that localizing an aircraft by ADS-B will function only as long as the transponder is working. A transponder failure or a manual deactivation due to an electrical problem will prevent Air Traffic Control from locating an aircraft in distress.

In 2008 the German Aerospace Center (DLR) started to investigate whether 1090ES ADS-B signals broadcasted by aircraft can be received on board of low earth orbiting (LEO) satellites [1]. The studies and simulations lead to the DLR project "ADS-B over Satellite" (AOS), which served for the development of a space-qualified ADS-B receiver and antenna system. The In-Orbit Demonstration (IOD) aimed at demonstrating the feasibility of worldwide satellite based ADS-B surveillance [2].

AOS is conducted in the frame of ESA's PROBA-V mission (PROBA Vegetation [3]) and was successfully launched on top of Europe's newest launcher VEGA on 7th of May 2013 at 04:06:31 CEST from the European spaceport Centre Spatial Guyanese (CSG) in French Guyana.

The IOD is capable to receive, decode and forward all Mode S downlink telegram formats. This includes the DF17 
Extended Squitter comprising ADS-B information and DF11 Short Squitter.

ADS-B over Satellite is the first experiment of its kind and a first step for demonstration and verification of space based air traffic surveillance.

\section{RECEIVER TECHNOLOGY}

Detection and demodulation of weak Mode S signals of Mode S-based ADS-B messages at distances of several hundred kilometers is not comparable to any other ADS-B application on ground or within airspace. According to the restrictive link budget there is only a small margin for a Mode $\mathrm{S}$ correlation and demodulation process to function properly. One must be aware that the hosted payload in LEO is seeking for very weak Mode S signals. Transponder signals have not been developed for received levels below $-90 \mathrm{dBm}$ in general and have poor correlation properties. Originally, Mode $\mathrm{S}$ telegram formats were designed to mitigate some severe SSR problems as Garbling and FRUIT. A conventional Mode S preamble correlation of baseband pulses does normally not bring up sufficient process gain for a successful detection of very weak signals.

The developed receiver makes use of the phase coherence between single telegram pulses [4]. In modern airliner transponders, the generated RF pulses origin from a single clock source and the signal is then amplified to match the required output level. Typically, the carrier frequency deviation from $1090 \mathrm{MHz}$ is below $100 \mathrm{kHz}$ so the received phase is a useful quantity to enhance the correlation process.

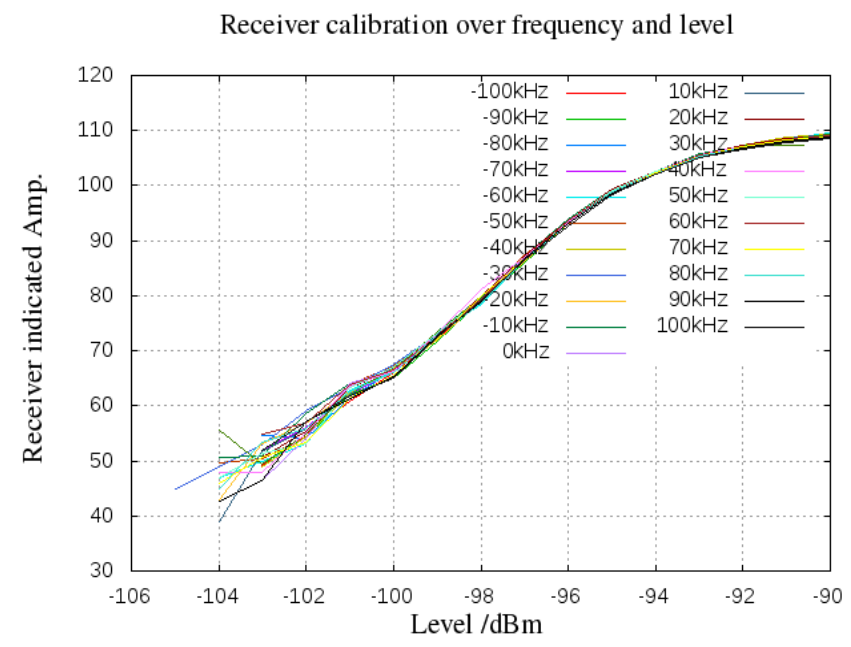

Fig. 1: Receiver calibration with different levels and frequency deviations from $1090 \mathrm{MHz}$

In Fig. 1 the receiver calibration results are shown using various test levels and frequency deviations from the nominal value $1090 \mathrm{MHz}$. As the receiver indicates an 8 Bit amplitude value for each received Mode $\mathrm{S}$ telegram, this allows tracing it back to the absolute signal level in $\mathrm{dBm}$ at the receiver's input. It can be seen that the receiver is decoding telegrams well below $-100 \mathrm{dBm}$ which is sufficient for catching weak transponder signals. Using a $10 \mathrm{kHz}$ step in calibration for frequencies within $+/-100 \mathrm{kHz}$ deviation around $1090 \mathrm{MHz}$, the curves show no significant variance. Therefore, an additional frequency dependency can be neglected.

A special feature is included in the receiver that allows to record and downlink the raw 16 bit intermediate frequency (IF) samples at full telegram length for a limited number of Mode $\mathrm{S}$ telegrams. This allows improving receivers and correlation algorithms in a lab having the original signal source from space in a box. This innovative technique will be tested and improved during mission runtime since the receiver FPGA configuration and the firmware of its embedded processor can be changed from remote.

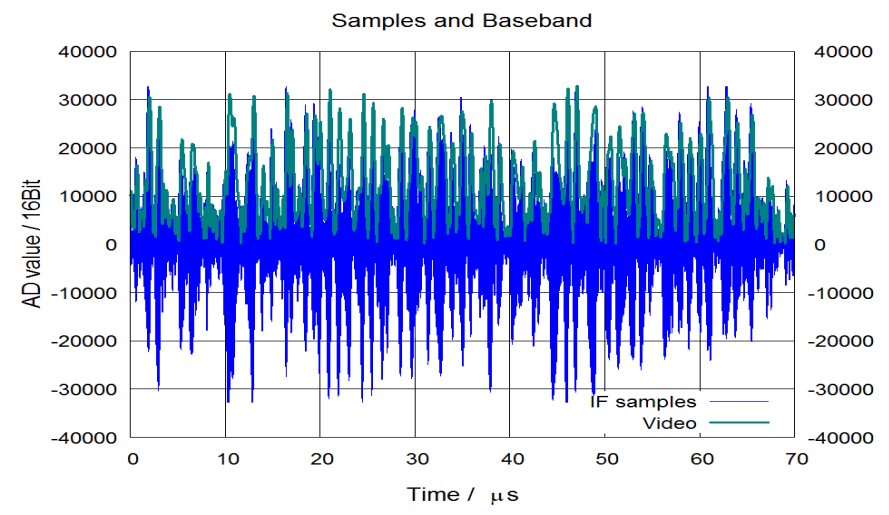

Fig. 2: SSR band pass raw data from IF

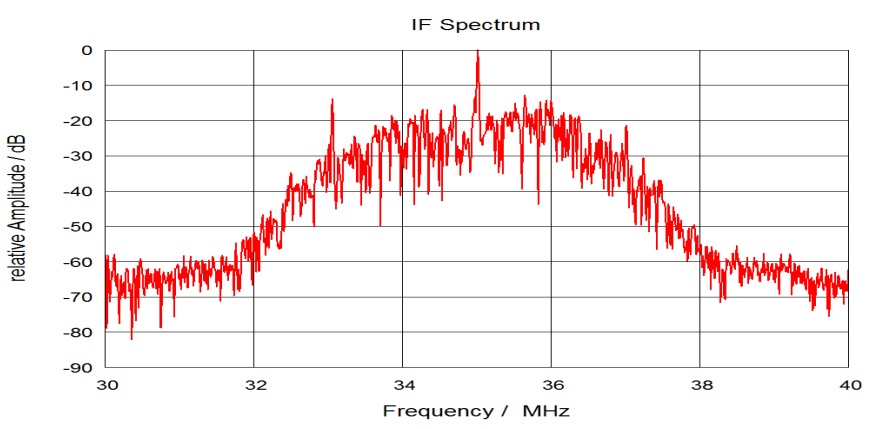

Fig. 3: SSR IF spectrum

In Fig. 2 an example of a DF11 Mode S Short Squitter at a level of a $-95 \mathrm{dBm}$ is given with plotted IF samples (band pass signal) and video pulses (baseband). Applying a transformation in the frequency domain, a spectrum as shown in Fig. 3 can be generated.

\section{RESULTS}

\section{A. General Aspects}

The evaluation of the results achieved should take into account, that the IOD is the first of its kind, and no comparable experiences have been made so far or evaluation results exist worldwide. Only a single satellite is equipped with a spacequalified ADS-B receiver, and due to limitations in costs and time but in particular resources available on PROBA-V concerning electrical power, equipment mass and geometry a relatively simple antenna and receiver design was implemented. 
The reception of 1090 Extended Squitter ADS-B messages on board of the PROBA-V satellite is mainly affected by the following issues, which can lead to a loss of ADS-B information:

- RF signal loss due to the long distance between the receiving satellite at an altitude of approximately 820 $\mathrm{km}$ and the transmitting aircraft at an altitude of 0 to 12 $\mathrm{km}$. In comparison the range for ground based ADS-B amounts to $150 \mathrm{NM}$.

- RF signal loss due to the shape of the vertical antenna pattern of the satellite and the aircraft antennas.

- Garbling, which means that messages arriving at the ADS-B antenna at the same time overlap and thus may not be decoded by the ADS-B receiver.

- The speed of the PROBA-V LEO satellite of about $27000 \mathrm{~km} / \mathrm{h}$, which leads to a time of observation for each detected aircraft limited to about 3 minutes maximum.

Additionally it must be considered, that the ADS-B receiver on board of PROBA-V is a hosted payload, whilst the main mission is the vegetation scanner. So the antenna mounting position was a compromise in order not to disturb the main mission and the other payloads.

\section{B. Link Budget}

As indicated in Section II, Mode S Transponder signals have not been developed for levels below $-90 \mathrm{dBm}$ in general and have poor correlation properties.

Fig. 4 shows the achieved correlation quality of the space based ADS-B receiver for the satellite pass 4119, recorded on the 6th of June 2014. Obviously, the better the signal power and neglecting the frequency deviation, the better the performance of the correlation algorithm is. The highest value measured in pass 4119 has its maximum ADC value around 110 and a minimum between 40 and 50 . These values can be transformed to signal power values of roughly $-90 \mathrm{dBm}$ as a maximum and -102 to $-104 \mathrm{dBm}$ as a minimum.

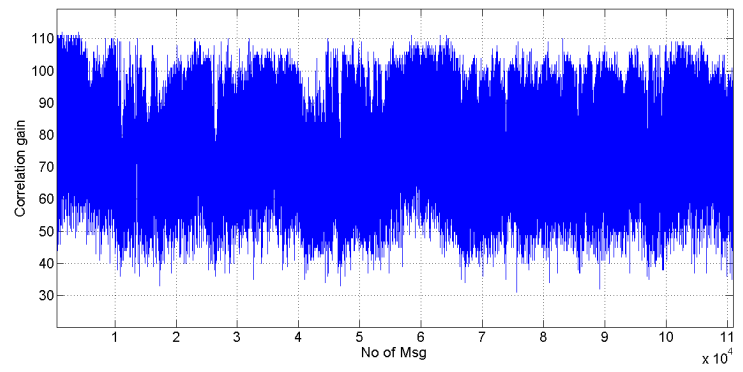

Fig. 4: Correlation Quality in pass 4119

Summarizing the power values over a period of one month as shown in Fig. 5 the dynamic bandwidth of the receiver is clearly visible with an absolute upper value of $-90 \mathrm{dBm}$ and an absolute minimum of $-104 \mathrm{dBm}$ measured at the receivers LNA. Thus, the actual correlation method yields to a receiver sensitivity of $-104 \mathrm{dBm}$.

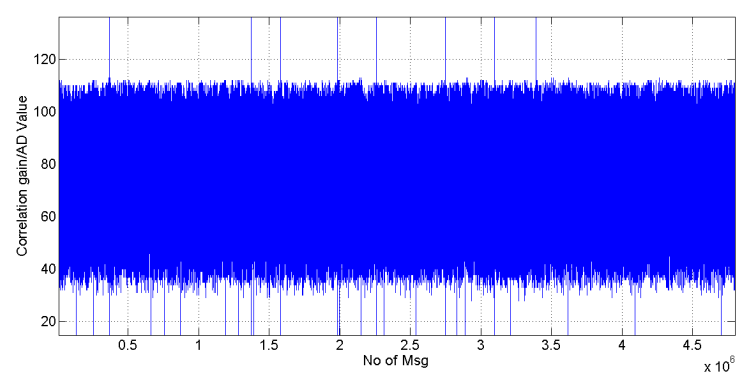

Fig. 5: Correlation quality in February 2014

During the development phase, when the orbital parameters of PROBA-V had not been disclosed yet, the calculation of the link budget was based on the following assumptions:

- $\quad$ Orbit Altitude $=780 \mathrm{~km}$

- Inclination $=98^{\circ}$

- $\quad$ Minimum Reception Distance $=780 \mathrm{~km}$

- $\quad$ Maximum Reception Distance $=2742 \mathrm{~km}$

The calculated power values of maximal $-89 \mathrm{dBm}$ and $-103 \mathrm{dBm}$ minimal were fully confirmed by the in-orbit measurements with ADS-B over satellite.

\section{Received and decoded Aircraft Positions}

In the following some examples of received and decoded aircraft tracks and position plots will be presented.

Fig. 6 shows several orbits of PROBA-V during pass 2699 on 11th February 2014 together with the recorded aircraft tracks. A pass starts after PROBA-V gets in the range of the of the satellite operation center Redu in Belgium, where data stored onboard is downlinked in a burst. The pass will end after several orbits when the satellite again gets near the ground station.

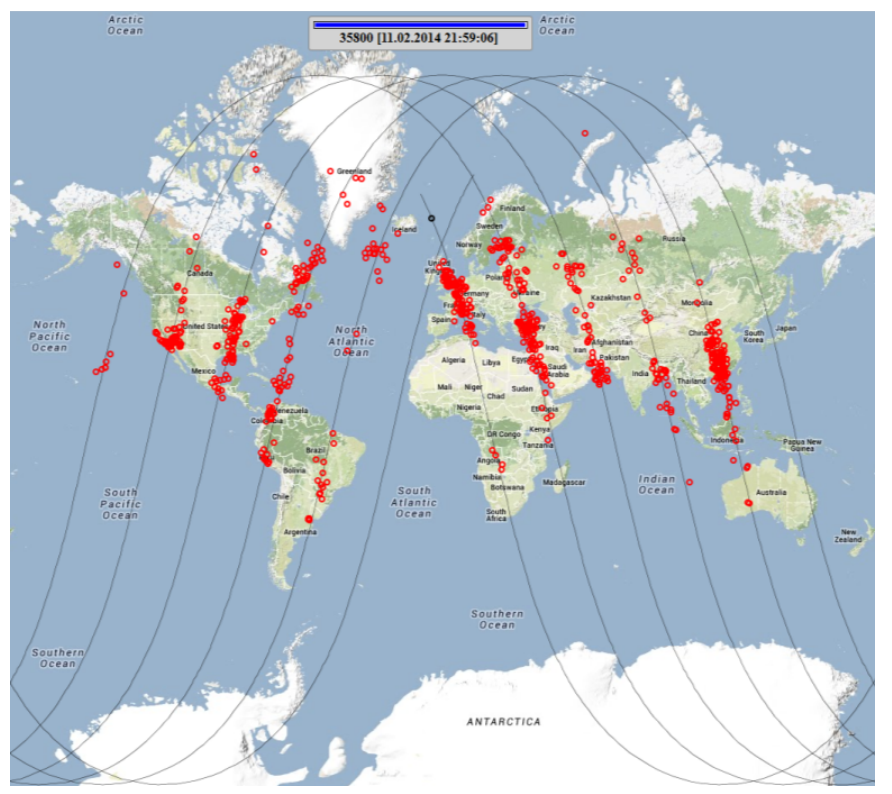

Fig. 6: Aircraft Tracks observed by PROBA-V 
The red dots represent track segments of aircraft detected by the satellite when its orbit passes them. With a size of the antenna footprint of approximately $1200 \mathrm{~km}$ along track and $500 \mathrm{~km}$ lateral, PROBA-V scans the global airspace strip by strip.

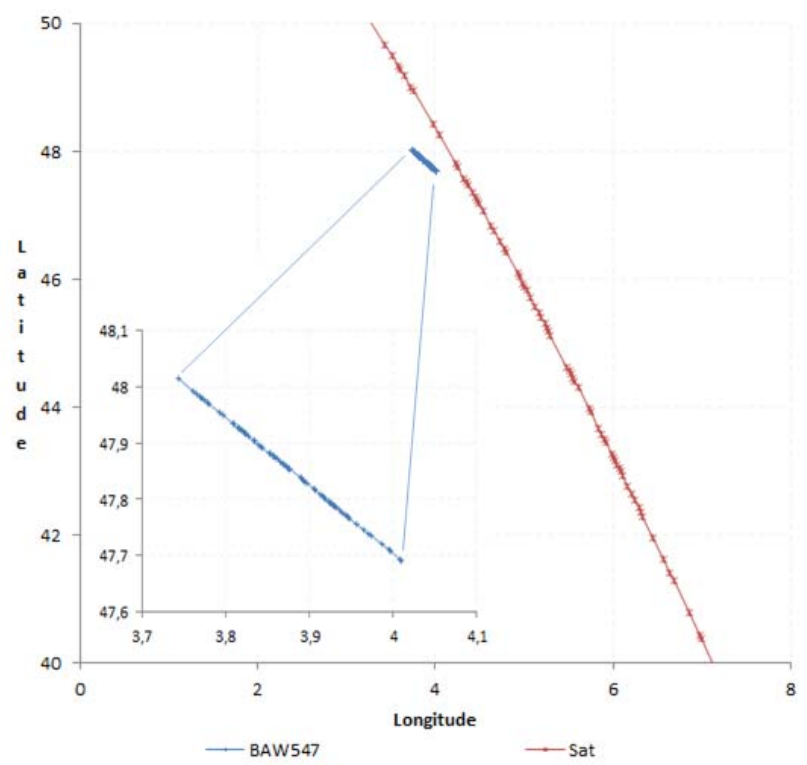

Fig. 7: Trajectory of a flight from Rome to London observed over France

Fig. 7 gives a more detailed view on a single trajectory. The example shows an aircraft flying over France from Rome to London. The blue line is the aircraft track with dots for every aircraft position. It shows several gaps, where no position messages were received. The red line is the ascending satellite flight path, and the red dots show the satellite positions corresponding to the received position messages. As can be seen the observation period for an aircraft is short in comparison to the length of the satellite's orbital segment.

Fig. 8 shows the same trajectory inside the footprint. The $\mathrm{x}-$ axis points in the satellite's track direction, with the positive values in front of nadir. The $y$-axis presents the lateral offset between the aircraft positions and the orbit.

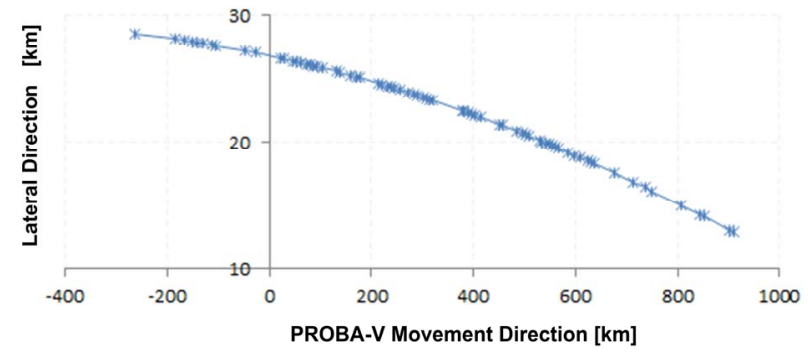

Fig. 8: Trajectory of the Flight within the Footprint

Fig. 9 shows the trajectory of an aircraft inside the footprint departing from Lijiang Airport in South China. The footprint has been clipped to a length of $+-600 \mathrm{~km}$. The green dots are surface positions of taxi movements and the take-off run, the blue dots show the airborne track. Obviously satellite-based surveillance can principally provide satisfying results also for ground movements of aircraft.

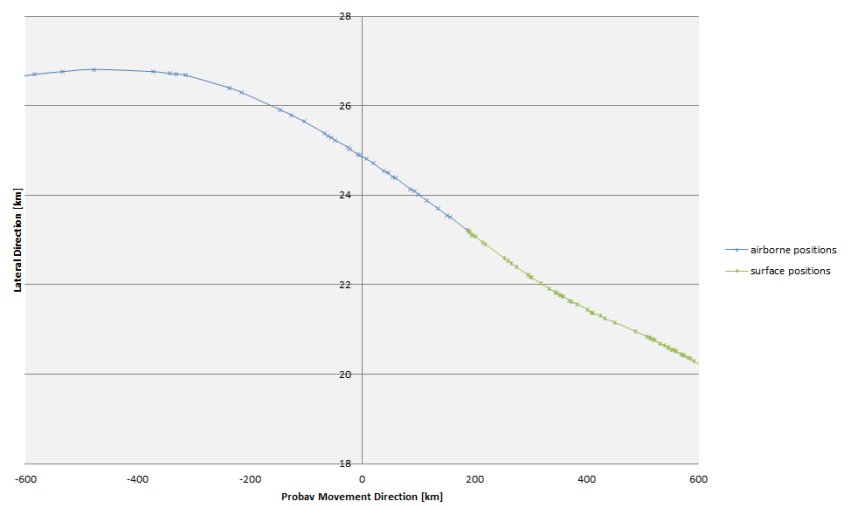

Fig. 9: Trajectory of an Aircraft departing from Lijiang Airport in the Antenna Footprint

\section{Antenna Footprint}

An essential aspect for the reception of ADS-B messages in space are the receiving conditions for the $1090 \mathrm{MHz}$ extended squitter signal on board of a satellite. The signal path between a LEO satellite orbiting at altitude of $820 \mathrm{~km}$ and an aircraft at an altitude between 0 and $12 \mathrm{~km}$ is much longer than between an aircraft and an ADS-B ground station. The resulting propagation loss requires that the Mode $\mathrm{S}$ signals have to be detected nearly at noise level by a correlation process.

The shape and extent of the footprint was determined by compiling histograms, which reflect the number of received messages with respect to the aircraft position in relation to the satellite position projected to an $\mathrm{x}-\mathrm{y}$ plane. Fig. 10 shows a histogram for decoded position messages.

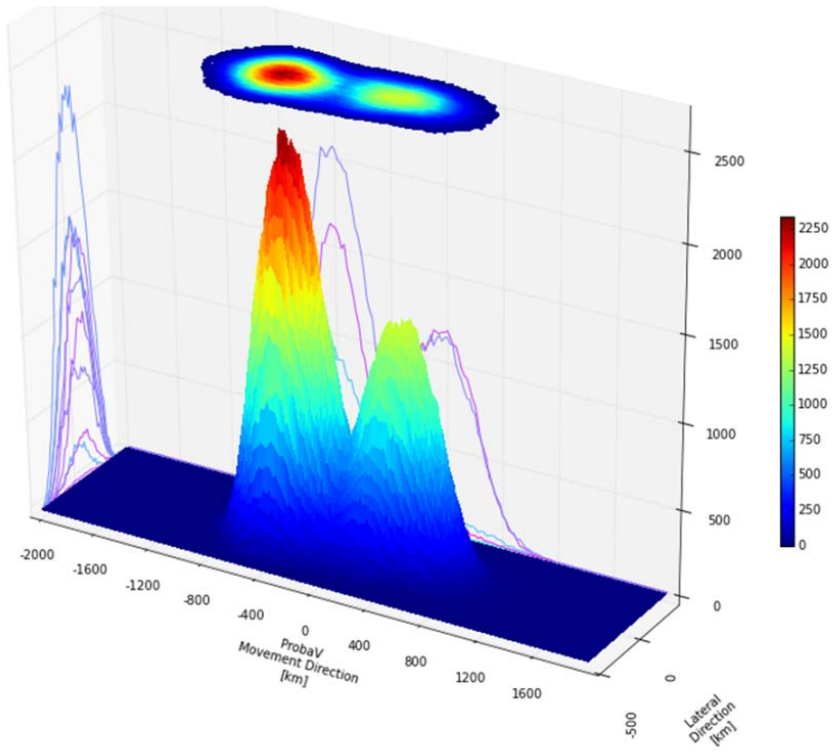

Fig. 10: Histogram of received Position Messages in the Antenna Footprint

The histogram shows two peaks, one lower in movement direction and the other higher behind the satellite, with a dip at the Nadir position. The footprint is not symmetrical, which is 
caused by a solar panel which protrudes over the front edge of the lower surface, the asymmetric mounting positions of the patch antennas and other equipment mounted at the underside of the satellite.

The peaks of the histogram can be explained by the antenna radiation patterns of the receiving antennas of the satellite and the transmitting antenna of the aircraft.

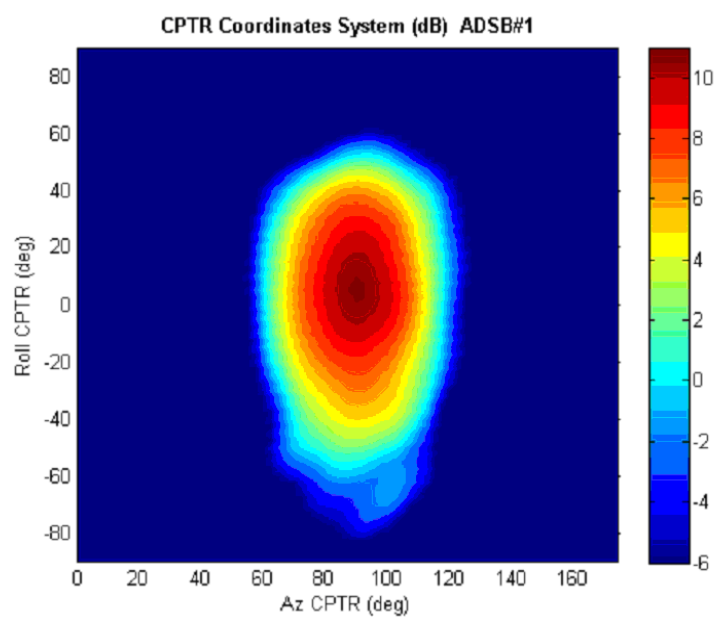

Fig. 11: Antenna Radiation Pattern of the PROBA-V ADS-B Antenna

Fig. 11 shows the measured radiation pattern of the ADS-B patch antennas mounted on the underside of PROBA-V. The resulting combined antenna radiation pattern has an overall oval shape in the direction of the satellite movement and the maximum sensitivity slightly behind the Nadir direction.

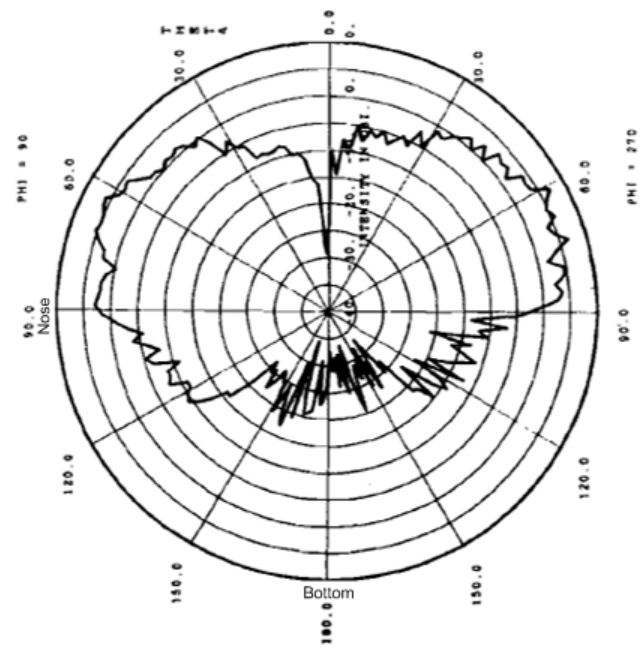

Fig. 12: Vertical Antenna Radiation Pattern of a top mounted L-Band Antenna [5]

Fig. 12 shows the vertical antenna radiation pattern of an aircraft ATC top antenna, from which the satellite will receive the ADS-B signals.

From the toroidal shape and the recess in the center results the so-called "cone of silence" vertically above an aircraft, so that an aircraft more or less directly below the satellite might not be detected at all or with a lower probability. The shape of the footprint strongly suggests that both "complementary" antenna patterns do not compensate each other, which results in the dip in the middle of the footprint.

\section{E. Performance Parameters}

The verification of space based 1090ES ADS-B surveillance required the identification of performance parameters, in accordance to existing requirements for ground based ADS-B surveillance given in the respective documents by EUROCAE and EUROCONTROL [6] [7] [8].

In the following some applicable performance parameters shall be shortly described.

a) Probability of Target Acquisition: As was seen from the comparison of reference traffic and detected aircraft tracks certain targets are not detected at all when passed by the satellite's antenna footprint. Thus a specific performance parameter for target detection has been defined:

The Probability of Target Acquisition (PTA) shall be defined as the percentage ratio between the actual number of targets detected and the expected number of targets to be detected within a certain area or time of observation. Detection means the provision of positional data.

\section{b) Probability of Detection:}

The probability of detection (POD) shall be defined as the percentage ratio between the actual number of position messages received and the expected number of position messages.

\section{c) Probability of Target Identification:}

The Probability of Target Identification (PTI) is the percentage ratio between the actual number of aircraft identified and the expected number of aircraft which should have been identified.

Aircraft normally will not change their identification (call sign) during flight, and thus a relatively sporadic identification will be sufficient, as by the ICAO address comprised in every ADS-B message each message can be allocated to a flight once identified.

\section{d) Probability of Identification:}

The probability of identification (POI) is the percentage ratio between the actual number of identification messages received and the expected number of identification messages.

\section{F. Evaluation of Performance Parameters}

For the evaluation of the performance parameters mentioned above, a zone filtering has been provided, which facilitates to filter the received ADS-B messages in accordance to defined areas on the earth map. This allows e.g. to mask out areas with high traffic density ("default") as is North- and South-America, Europe and Asia and thus a high risk of garbling und therefor non-decodable messages. On the other hand the filtering allows for the performance evaluation for 
certain areas, like north Atlantic or Pacific transoceanic flight routes. The performance parameters shown in the Tables I to III have been computed from ADS-B messages received during March 2014.

TABLE I: ProbabILITY OF TARGET ACQUISITION AND PROBABILITY OF TARGET IDENTIFICATION

\begin{tabular}{|lrrrrr|}
\hline \multicolumn{1}{|c}{$\begin{array}{c}\text { Zone } \\
\text { Filter }\end{array}$} & $\begin{array}{r}\text { Aircraft } \\
\text { detected }\end{array}$ & $\begin{array}{r}\text { Aircraft } \\
\text { expected }\end{array}$ & PTA [\%] & $\begin{array}{r}\text { Aircraft } \\
\text { identified }\end{array}$ & PTI [\%] \\
\hline All & 61798 & 64652 & 95,6 & 44665 & 69,1 \\
\hline Australia & 2426 & 2559 & 94,8 & 1790 & 69,9 \\
\hline Default & 23943 & 30719 & 77,9 & 16578 & 54 \\
\hline East Asia & 12743 & 13859 & 91,9 & 9142 & 66 \\
\hline Europe & 15106 & 17235 & 87,6 & 9538 & 55,3 \\
\hline $\begin{array}{l}\text { North } \\
\text { Atlantic }\end{array}$ & 5039 & 6201 & 81,3 & 3480 & 56,1 \\
\hline Pacific & 997 & 1301 & 76,6 & 734 & 56,4 \\
\hline
\end{tabular}

TABle II: Probability OF DETECTION

\begin{tabular}{|lrrr|}
\hline \multicolumn{1}{|c}{ Zone Filter } & $\begin{array}{l}\text { Positions } \\
\text { detected }\end{array}$ & $\begin{array}{l}\text { Positions } \\
\text { expected }\end{array}$ & POD [\%] \\
\hline All & 1464447 & 10878627 & 13,5 \\
\hline Australia & 95203 & 425298 & 22,4 \\
\hline Default & 520709 & 3376666 & 15,4 \\
\hline East Asia & 270910 & 2102401 & 12,9 \\
\hline Europe & 244345 & 2290756 & 10,7 \\
\hline North Atlantic & 92458 & 780939 & 11,8 \\
\hline Pacific & 20508 & 156205 & 13,1 \\
\hline
\end{tabular}

Table III: Probability of Identification

\begin{tabular}{|l|r|r|r|}
\hline \multicolumn{1}{|c|}{ Zone Filter } & \multicolumn{1}{|c|}{$\begin{array}{c}\text { Ident } \\
\text { Messages } \\
\text { detected }\end{array}$} & $\begin{array}{c}\text { Ident } \\
\text { Messages } \\
\text { expected }\end{array}$ & POI [\%] \\
\hline All & 149568 & 1087863 & 13,7 \\
\hline Australia & 7240 & 42530 & 17,0 \\
\hline Default & 50641 & 337667 & 15,0 \\
\hline East Asia & 29439 & 210240 & 14,0 \\
\hline Europe & 24863 & 229076 & 10,9 \\
\hline North Atlantic & 8887 & 78094 & 11,4 \\
\hline Pacific & 2131 & 15621 & 13,6 \\
\hline
\end{tabular}

In general it must be said that the performance parameters of the experimental system at hand do not fulfill the requirements of an operational surveillance system. This can be explained mostly be the influence of the antenna diagrams of the ADS-B antenna on board of PROBA-V, pointing vertically down in the cone of silence of the aircraft ATC antenna or its vicinity.

Better results could be expected by a slant pointing satellite ADS-B antenna, but which could not be mounted on PROBAV due to the restrictions for the hosted payload. For the future it is planned to operate PROBA-V at a slant attitude during periods, when it passes the dark side of the earth and the vegetation scanner thus is not in operation. With this configuration the antenna will point laterally to the aircraft antenna in the vicinity of the radiation pattern maximum.

\section{SUMMARY}

The PROBA-V in-orbit demonstration has proven, that space based 1090ES ADS-B surveillance is technically feasible, and thereby the goals of the project "ADS-B over Satellite" have been attained.

Though the experimental equipment used in the IOD does not provide the performance required for an operational system, essential results could be achieved and conclusions be drawn for the design of a future operational system. A space based ADS-B surveillance system providing worldwide coverage will consist of a fleet of satellites equipped with highly sophisticated multi-channel ADS-B receivers. Slantwise pointing spot beam antennas for all channels will overcome the cone of silence of aircraft transponder antennas, avoid a signal overload of the single receiver channels and provide an ample overall footprint for each satellite.

\section{ACKNOWLEDGMENT}

This project was jointly funded by the DLR Directorates of Space and Aviation as well as ESA's General Support Technology Programme (GSTP). The Authors would like to thank the ESTEC TEC Department and German Space Agency for their technical and personal support.

\section{REFERENCES}

[1] T. Delovski, L.-C. Hauer and J. Behrens, "ADS-B high altitude measurements in non-radar airspaces.," European Journal of Navigation, 2010.

[2] T. Delovski, K. Werner, T. Rawlik and J. Behrens, "ADS-B over Satellite - The worlds first ADS-B receiver in Space," in Small Satellite Systems and Services Symposium, Porto Pedro, Spain, 2014.

[3] S. Blair, V for vegetation: The mission of Proba-V., Noordwijk: ESA ESTEC, 2013.

[4] J. Bredemeyer, ,Secondary signal receiver and method for determining defined receiver times of secondary radar signals in a multilateration system," European Patent EP000002280290, 2011.

[5] J. S. K. J. Keeping, "Scale model Pattern Measurements of Aircraft LBand Beacon Antennas, Project Report ATC-74," Massachusetts Institute of Technology, Lexington, Massachusetts, 1975

[6] Safety, Performance and Interoperability Requirements Document for ADS - B - NRA application, ED-126, EUROCAE, 2006.

[7] Minimum Operational Performance Standards for $1090 \mathrm{MHz}$ Extended Squitter Automatic Dependent Surveillance - Broadcast (ADS - B) and Traffic Information Services - Broadcast (TIS - B), ED-102A, EUROCAE, 2009.

[8] EUROCONTROL Standard Document for Radar Surveillance in enroute Airspace and major Terminal Areas, EUROCONTROL, 1997. 